interaction between calendar time, age and treatment (Likelihood Ratio Test $\mathrm{p}<0.0001)$. Stratum specific estimates of the increase in incidence for each age group from 2000 to 2013 showed the greatest increase, 49\%, in those aged under 45 years who were treated conservatively without surgery (Incidence Rate Ratio 1.49, 95\% CI 1.36-1.61) adjusting for sex and region. This increase was seen in conjunction with an increased use of diagnostic imaging especially in the younger age groups. One year survival was highest in those under the age of 65 years who were treated conservatively (96.4\%). In those over 65 years one year survival was lower and varied as to whether patients were treated operatively or not (71.4\% vs. 50.7\%).

Conclusions The incidence of perforated diverticular disease has increased from 2000 to 2013 with the greatest increase in younger age groups who also had the best survival. The increase in incidence in younger groups in part may be due to the identification of patients with localised perforations more frequently identified due to an increase in the use of CT scans.

\section{PTU-070 INCIDENCE OF AND SURVIVAL FOLLOWING DIVERTICULAR ABSCESS: A POPULATION-BASED COHORT STUDY FROM ENGLAND}

\begin{abstract}
${ }^{1,2,3}$ Professor Joe West ${ }^{*},{ }^{1,2,3}$ Colin Crooks, ${ }^{4}$ Harmony Otete, ${ }^{1,2,3}$ David Humes. ${ }^{1}$ Division of Epidemiology and Public Health, University Of Nottingham, Nottingham, UK; ${ }^{2}$ NIHR Nottingham Biomedical Research Centre, Nottingham, UK; ${ }^{3}$ Notttingham Digestive Diseases Centre, Nottingham, UK; ${ }^{4}$ School of Pharmacy, University of Nottingham, Nottingham, UK
\end{abstract}

\subsection{6/gutjnl-2018-BSGAbstracts.411}

Background Diverticular abscess represents a significant complication of diverticular disease. We aimed to provide population based estimates of the incidence of diverticular abscess and assess variation by age, calendar time, treatment and sex. Methods We undertook a historical cohort study using linked primary and secondary care data from 2000-2013 from England. We identified cases of diverticular abscess older than 18 years, calculated incidence rates, and modelled variation using Poisson regression along with estimating one year survival with life tables.

Results We identified 622 cases with an overall incidence of 1.9 per 100000 person years (pyrs) (interquartile range (IQR) 1.7-2.0 per 100000 pyrs). Over the time period of the study there was a 1.5 fold increase in the incidence of diverticular abscess (adjusted IRR 1.5, 95\% CI 1.0-2.5). This increase was seen in conjunction with an increased use of diagnostic imaging with $92.2 \%$ having a CT in 2013 compared to $60 \%$ in $2007(\mathrm{p}=0.003)$. One year survival was $80.1 \%$ (95\% CI, 76.7\%-83.1\%) overall and was lowest in those undergoing surgery $68.7 \% \%$ (95\% CI 60.3\%-75.7\%).

Conclusions There has been a 1.5 fold increase in the incidence of diverticular abscess from 2000 to 2013. The condition is associated with a poor one year survival especially following surgery. This increase in incidence may in part be due to improved identification of cases due to an increased use of imaging.
PTU-071 RISK STRATIFICATION OF SYMPTOMATIC PATIENTS USING FAECAL BIOMARKERS AND URINARY VOLATILE ORGANIC COMPOUNDS

1,2 Monika Widlak*, ${ }^{3}$ Matthew Neal, ${ }^{4}$ Emma Daulton, ${ }^{5}$ Claire Thomas, ${ }^{5}$ Claudia Tomkins, ${ }^{6}$ Baljit Singh, ${ }^{7}$ Chris Harmston, ${ }^{4}$ Alfian Wicaksono, ${ }^{7}$ Charles Evans, ${ }^{5,8}{ }^{1}$ teve Smith, ${ }^{3}$ Richard Savage, ${ }^{4}$ James Covington, ${ }^{1,2,9}$ Ramesh Arasaradnam. ${ }^{1}$ Department of Gastroenterology, University Hospitals Coventry and Warwickshire NHS Trust, Coventry, UK; ${ }^{2}$ Medical School, University of Warwick, Coventry, UK; ${ }^{3}$ Department of Statistics, University of Warwick, Coventry, UK; ${ }^{4}$ School of Engineering, University of Warwick, Coventry, UK; ${ }^{5}$ Department of Biochemistry, University Hospitals Coventry and Warwickshire NHS Trust, Coventry, UK; ${ }^{6}$ Department of Colorectal Surgery, Leicester General Hospital, Leicester, UK; ${ }^{7}$ Department of Colorectal Surgery, University Hospitals Coventry and Warwickshire NHS Trust, Coventry, UK; ${ }^{8}$ Midlands and North West Bowel Cancer Screening Programme Hub, Rugby, UK; ${ }^{9}$ Applied Biological and Experimental Sciences, University of Coventry, Coventry, UK

\subsection{6/gutjnl-2018-BSGAbstracts.412}

Introduction There remains an urgent need for non-invasive, low cost methods for diagnosis of colorectal cancer (CRC). We undertook a diagnostic accuracy study using faecal haemoglobin (F-Hb), faecal calprotectin (FCP) and urinary volatile organic compounds (VOCs) in patients presenting with lower gastrointestinal symptoms referred via Two Week Wait colorectal pathway.

Methods Of 1850 patients approached, 1016 were recruited prospectively. Of these, 562 with complete colonic investigations returned matched urine and stool samples and were included in the final statistical analysis.

Results The sensitivity and specificity for CRC using F-Hb were 0.80 (95\% confidence interval (CI): $0.66-0.93)$ and 0.93 (CI: 0.91-0.95) respectively. The negative predictive value (NPV) was 0.99 (CI: 0.98-1.0). Using urinary VOCs the sensitivity and specificity were 0.63 (CI: $0.46-0.79$ ) and 0.63 (CI: $0.59-0.67$ ) respectively and the NPV was 0.96 (CI: 0.94 0.98). For those with F-Hb negative CRC (false negatives), adding urinary VOCs revealed the sensitivity of 0.97 (CI: $0.90-1.0)$ and specificity of 0.72 (CI: 0.68-0.76) with the NPV of 1.0 (CI: 0.99-1.0).

Conclusions Urinary VOCs applied to a F-Hb negative group excludes CRC with the NPV of 1.0. Thus, the addition of urinary VOCs shows promise as a second stage test in investigating symptomatic population.

\section{PTU-072 BREATH TESTING FOR COLORECTAL POLYPS AND CANCER- A LOAD OF HOT AIR?}

${ }^{1,2}$ Georgia Woodfield*,${ }^{1}$ llaria Belluomo, ${ }^{1}$ Gengping Lin, ${ }^{1,2}$ Piers Boshier, ${ }^{1}$ Andrea Romano, ${ }^{2} J o h n$ Martin, ${ }^{3}$ Chris Groves, ${ }^{4}$ Laura Marelli, ${ }^{5}$ Brian Saunders, ${ }^{1}$ Wendy Atkin, ${ }^{1,2}$ George B Hanna. 'Imperial College, London, UK; ${ }^{2}$ Imperial College Healthcare NHS Trust, London, UK; ${ }^{3}$ St George's Healthcare NHS Trust, London, UK; ${ }^{4}$ Homerton University Hospital NHS Foundation Trust, London, UK; ${ }^{5}$ St Mark's Hospital, London, UK

\subsection{6/gutjnl-2018-BSGAbstracts.413}

Introduction Colorectal cancer (CRC) is the 2nd most common UK cause of cancer death. The bowel cancer screening programme (BCSP) targets those aged $60-74$, but is dependent upon uptake. Some colonoscopies may be unnecessary and are not without risk. A breath test could be a useful 